\title{
An Evolutionary Mono-Objective Approach for Solving The Menu Planning Problem
}

\author{
Rafaela P. C. Moreira*, Elizabeth Wanner ${ }^{\dagger}$, Flávio V. C. Martins ${ }^{\ddagger}$, João F. M. Sarubbi ${ }^{\S}$ \\ *PPGMMC - CEFET-MG, Brazil - rafapcmor@gmail.com \\ ${ }^{\dagger}$ EAS - Aston University - UK; PPGMMC - CEFET-MG - Brazil - e.wanner@aston.ac.uk \\ ${ }^{\ddagger}$ PPGMMC - CEFET-MG - Brazil - flaviocruzeiro@decom.cefetmg.br \\ §Departamento de Computação- CEFET-MG - Brazil - joao@decom.cefetmg.br
}

\begin{abstract}
This work proposes an evolutionary approach to solve the Menu Planning Problem. Our work uses the Brazilian school context and our principal goal is to create menus that minimize the total cost of these menus. However, those menus must also satisfy requirements of the Brazilian government, such as: (i) student age group, (ii) school category, (iii) school duration time, (iv) school location, (v) variety of preparations, (vi) harmony of preparations, (vii) maximum amount to be paid for each meal and, (viii) lower and upper limits of macronutrients. The results demonstrate that the evolutionary approach is not only able to generate a set of inexpensive and healthy menus but also respect the required set of constraints. A constrained deterministic approach is performed to generate 5-day menu through a greedy-based function taking into account the normalized sum of all macronutrients and the monetary cost of the menu. A comparison between the 5-day menu obtained by the proposed approach and the constrained greedy-based approach menu is carried out. Despite the fact the obtained menu outperforms the greed-based menu taking into account the total cost, this difference is not so expressive. However, all macronutrients were outside the pre-defined range at least in one day of the week. The 5-day menu obtained by the proposed approach is evaluated by a nutritionist. The overall quality of the menu is outstanding and the time spent to generate it is 60 seconds.
\end{abstract}

Index Terms-Menu Planning; School Feeding; Evolutionary Computation; Single-objective optimization

\section{INTRODUCTION}

Changings on human diet, physical activity patterns, and nutritional status affect the nutritional outcomes such as average stature and body composition. This nutrition transition is affected by economic and demographic changes. Many countries suffer a nutritional transition developing diets high in sugar and fat. A scenario that used to be of malnutrition now becomes overweight and obesity. A survey conducted by the Institute for Health Metrics and Evaluation [1] showed that, from 1980 to 2013, obesity and overweight together increased $27.5 \%$ among adults and $47.1 \%$ among children. No country has had a significant reduction in the obesity rates in the last 33 years. Obesity is considered a worldwide epidemic. According to [2], about 2.1 billion people in the world suffer from overweight or obesity, equivalent to approximately $30 \%$ of the world's population and this percentage can rise to half the world's inhabitants in 2030.

According to WHO [3], there are 40.6 million obese children worldwide under the age of five in 2016. In Brazil, according to IBGE, about one-third of the population between five and nine years old is overweight and, if no measures are taken, numbers will continue to rise [4]. The primary cause of this obesity epidemic is the increase in the supply of energy foods, with many calories, highly processed industrialized foods. These types of food, being tasty, cheap and widely advertised, facilitate overconsumption [3].

There is an urgent need for public health measures to prevent the obesity and, consequently, to save societal money. Having that in mind, the Brazilian government treats food security as a priority in the Pluriannual Plan 2016-2019 [5], which consists in a set of actions seeking to guarantee the right of a permanent and regular access to healthy feeding, in a sufficient quantity, without compromising the access to other essential needs. With the objective of changing this scenario of overweight and obesity, the government has invested in school feeding through the National School Feeding Program (PNAE). The PNAE offers not only school feeding but also food and nutritional education actions to students of all stages of basic education (nursery, primary school, high school and youth and adult education) in state and philanthropic schools and community entities (with the public power) [6].

Menu Planning Problem is a new version of classic Diet Problem combining nutritional and economic objectives with pre-defined requirements. This problem intends to find the best combination of items that attends the objectives and the imposed requirements [7].

For many children, the school meal they get is the only healthy food they eat all day. School meals provide access to various nutritious food promoting students' health. The Menu Planning is an important attribution of the nutritionist. She should prepare the school menu, respecting local and cultural eating habits, meeting specific nutritional needs, according to minimum percentages established in Resolution 26/2013 [6]. Attending the pre-defined requirements, such as variety, harmony of preparations and cost of the meal, is a difficult and time-consuming task.

With the goal of facilitating the work and contributing to the Menu Planning time reduction of, we propose the application of some computational intelligence techniques to elaborate the menus in an automatic way. The approach takes into account the age group, the daily nutritional needs of the students according to the category, age, location (urban, indigenous) 
and the length of school day, type of food (breakfast, morning snack, lunch, afternoon snack and dinner), variety, harmony of the preparations and cost of the meal.

In this paper, we consider two fundamental aspects: reducing the cost of the menu while meeting the daily nutritional needs of the students, taking into consideration the variety, color, consistency of the culinary preparations, the financial cost limit and lower and upper limits of macronutrients. A Genetic Algorithm is developed and applied to solve the Menu Planning Problem. The results show that, in 60 seconds, an inexpensive menu can be obtained. The menu also complies with the restrictions imposed by the Brazilian authorities.

This paper is organized as follows: Section II presents related works. Section III presents the problem definition. Section IV describes the applied methodology. Section V describes the results. And, finally, Section VI concludes the work.

\section{RELATED WORKS}

The literature shows various papers related to the Menu Planning Problem (MPP). In 1964, Balintfy [7] developed the first computer-aided menu planner with the goal of finding the minimum cost and satisfying the nutritional needs with a desired degree of variety, color and consistency. The author applied a Linear Programming technique and managed to reduce up to $30 \%$ of the cost of menus used in a hospital.

MenuGene is an automatic menu generator, developed by Gaal, Vassányi and Kozmann [8], which was used Genetic Algorithms (GA's) to prepare daily and weekly menus for Internet users. Constraints on the amount of carbohydrate, fats, and proteins according to age, sex, body mass, type of activities, and diseases of the person, in addition to food harmony can be added to the problem. The method was able to generate the menus respecting the nutritional requirements.

Kahraman and Seven [9] developed a program to solve the diet bi-objective problem using Genetic Algorithms and Weighted Sum approach, to present to the user a combination of healthy dishes, minimizing the cost and maximizing the total classification of the dishes. The user needs to inform the age and sex and sort preferred dishes on a scale of 1 to 10. They applied the algorithm to Multidimensional Knapsack Problem samples from a single small-scale goal and had achieved the optimal solution for them.

GIGISim (Glucose-Insulin and Glycemic Index Web Simulator) [10] is another Genetic Algorithm-based tool developed to prepare meals for Diabetics. The main goal was to supply the nutritional needs, satisfying taste preference, lifestyle and controlling blood glucose. Optimization tool reported back combinations of products and meals which cause the lowest possible glucose rise, suggest adding certain amounts of fats to each meal and vegetable fats instead of saturated ones.

Seljak [11] used an NSGA-II multiobjective algorithm to solve the problem of Menu Planning for hospital patients, aiming at minimizing costs and meeting nutritional needs, considering the functionality, seasonal evaluation, cost, flavor, consistency, color, temperature, form and method of preparation. The author modeled four objective functions and sixteen constraints. The algorithm was able to find solutions that met the established requirements.

The Pro-Diet [12] is a system which proposed the prescription of menus and the formulation of diet, intended to meet the principles of healthy eating, taking into account the variety of colors and meal, the combination of flavors, texture and the supply of all nutrients, in addition to patient data, such as age, sex, height and weight. Pro-diet was also based on GA's to find satisfactory solutions from a database already registered, allowing meals such as breakfast, lunch, snack, and dinner. The Pro-Diet served the purpose of generating menus quickly.

Cruz [13] proposed the problem of diet in day-care centers through Integer Linear Programming and solved in a multiobjective way. For the solution, the Convex Weighted Sum of the objective functions was used. The objectives considered were: to minimize costs and maximize levels of proteins, vitamins $\mathrm{A}$ and $\mathrm{C}$, iron, and calcium. Solutions needed to meet at least $70 \%$ of daily nutritional needs. As a result, the algorithm found 18 efficient solutions.

In Moreira et al. [14], a multiobjective approach for the Menu Planning Problem was proposed for the Brazilian school context. The goal was to, simultaneously, minimize the total cost and the nutritional error according to the Brazilian reference. The problem was solved via two formulations: (1) using the Weighted-Sum approach, the multiobjective problem was transformed into a single-objective problem and solved using a Genetic Algorithm, (2) using an evolutionary multiobjective algorithm, NSGA-II, for optimizing the objective simultaneously. The results showed the multiobjective approach is five times faster, with more non-dominated solutions.

It is important to highlight the differences between the proposed approach and the others available in the literature. Balintfy [7], Seljak [11], and Cruz [13] have as the main goal the generation of optimized and collective menus like the proposed approach. However, in this work, we use a different problem modelling, reducing the number of functions and avoiding some fragilities presented in the aforementioned works, such as the high number of constraints and a component based on subjectivity. The general structure of the menus and some constraints used in this work are based on Gomes [12]. Nonetheless, in [12], the focus is on individual dietary plans while in this work, the focus is on collective menus. Although Kahraman [9] models the problem in using a bi-objective approach and solves it using a Genetic Algorithm, the evaluation of the solutions takes into account personal data, unlike this proposed approach.

The difference between this proposed approach and the one presented in [14] lies in the formulation of the nutritional goal. While the former takes into account the total nutritional error, calculating the difference between the total of nutrients (macro and micronutrients), and the pre-defined ones, the 
latter aims to target the macronutrients into a pre-established range. The idea behind this is to avoid the under (or over) estimation of one or more nutrients when they are combined into only one nutritional function. Furthermore, targeting the macronutrients into a range, we expect to achieve a corresponding micronutrient target level.

\section{PRoblem DEFINITION}

Mathematically, the Menu Planning Problem (MPP) is an easy problem to formulate, but it belongs to the NP-Complete class [15]. Problems of this class are difficult to solve by exact deterministic techniques in polynomial time [11].

Since the MPP is combinatorial in nature, it is possible to determine how much time it would take to combine all possible preparations. Considering three meals (breakfast, lunch, and snack) for five days, and considering an operation is performed in $10^{-8}$ seconds in a standard computer and that the database has the following culinary preparations: rice (9) and beans (6); entree (29); side dish (15); main dish (12); dessert: fruits (11) and sweets (5); drink: juice (9) and milk or derivatives (5); bread/other cereal (10), there are about $1.719 \times 10^{14}$ possible menu combinations. Considering one day has 86400 seconds, this would take approximately 20 days to make all possible combinations.

In this paper, we want to create a five-day menu meeting the nutritional needs of 4-5 year-old students who study full-time according to the nutritional reference of the PNAE. Students in a full-time school day must get three or more meals representing at least $70 \%$ of their daily nutritional needs. Table I shows the recommended values for $70 \%$ of daily nutritional needs (DNN) for preschool students [6]. In this table, CHO refers to carbohydrates, PTN to proteins, and LIP to lipids. Other references can be found in [6]. These references are based on Food and Agriculture Organization of United Nations (FAO) [16].

Table I

REFERENCE VALUES FOR ENERGY, MACRO AND MICRO NUTRIENTS FOR PRESCHOOL (4 TO 5 YEARS)- 70\% DNN

\begin{tabular}{|c|c|c|c|c|c|c|c|c|}
\hline Energy & CHO & PTN & LIP & Fibers & \multicolumn{4}{|c|}{ Minerals $(\mathrm{mg})$} \\
\hline (Kcal) & $(\mathrm{g})$ & $(\mathrm{g})$ & $(\mathrm{g})$ & $(\mathrm{g})$ & $\mathrm{Ca}$ & $\mathrm{Fe}$ & $\mathrm{Mg}$ & $\mathrm{Zn}$ \\
\hline 950.00 & 154.40 & 29.70 & 23.80 & 17.50 & 560.00 & 7.00 & 91.00 & 3.50 \\
\hline
\end{tabular}

An important question that arrives is: if an individual consumes this diet in amounts that will satisfy energy needs, is it possible to say that the concentration of nutrients will be high enough to meet the nutrient needs? Having that in mind, ranges for the macronutrients (carbohydrates, proteins, and lipids) can be established and be used as indices of dietary quality. The FAO/WHO [17] establishes lower and upper macronutrient limits in relation to energy need. These ranges are given by Table II.

Let $V_{p}$ be the percentage value of the macronutrient, $V_{e}$, the reference energy value, in $\mathrm{kcal}$, and $k_{i}$ the energy value, in $\mathrm{kcal}$, of each macronutrient $(\mathrm{CHO}=4 \mathrm{kcal}, \mathrm{PTN}=4 \mathrm{kcal}$, and $\mathrm{LIP}=9 \mathrm{kcal})$, and $v_{i}$, the absolute value of each macronutrient.
Table II

LOWER AND UPPER LIMITS (\%) OF MACRONUTRIENTS: CHO, PTN, LIP

\begin{tabular}{|l|l|l|l|}
\hline & CHO & PTN & LIP \\
\hline LOWER & $55 \%$ & $10 \%$ & $15 \%$ \\
\hline UPPER & $75 \%$ & $15 \%$ & $30 \%$ \\
\hline
\end{tabular}

The absolute values, in grams, for each macronutrient is given by Equation (1):

$$
v_{i}=V_{e} \times V_{p} / k_{i}
$$

In this work, each day is composed of three meals: (i) breakfast; (ii) lunch; and, (iii) afternoon snack. Each meal has some preparations, according to its type. The breakfast is composed of three preparations: (i) bread or other cereal; (ii) milk or derivatives; and, (iii) fruit. The lunch is composed of seven preparations: (i) rice; (ii) beans; (iii) entree: salads and soups; (iv) side dish; (v) main dish; (vi) dessert: fruit or sweets; and, (vii) juice. The snack is composed of three preparations: (i) bread or other cereal; (ii) drink (milk or derivatives or juice); and, (iii) fruit.

The principle of PNAE is to promote healthy and adequate school meals in accordance to the age group, among other characteristics. Aiming to attend the PNAE principles, some constraints are introduced in the menu. These constraints deal with some qualitative aspects of the preparations such as (i) color; (ii) consistency, and (iii) variety. Other qualitative constraints such as (i) the cost limit sum for each meal for each student and (ii) lower and upper limits of the macronutrients are also introduced in the problem.

The qualitative constraints of the problem are described below:

- Color: A colorful meal provides a balanced and nutrient-rich dietary and also ensuring high intake levels of vitamins and minerals. For this work, aiming for different colored foods, we defined four predominant colors: (i) yellow; (ii) red; (iii) green; and, (iv) brown. This constraint is checked only for lunch and for the following types of preparations: (i) entree; (ii) side dish; (iii) dessert; and, (iv) drink, as directed by [12] and, to promote a colorful meal, the number of repetition of the same color in a given preparation should be less than or equal to 2 .

- Consistency: Food quality also has some subjective aspects. The texture is a largely subjective attribute and transmits confidence in the quality and acceptability of food. Two texture categories are used in this work: (i) liquid/pasty; and, (ii) semi-solid/solid. This constraint ensures that no more than one type of preparation classified as liquid/pasty can be presented in the lunch. The preparations checked are: (i) beans; (ii) entree; (iii) side dish; and, (iv) main dish.

- Variety: Eating many different foods helps maintain a healthy diet providing all vital nutrients to the body. Furthermore, choosing a variety of foods makes meals interesting. For providing that meals are varied, we check 
the number of repeated preparations in the menu. We analyze the repetitions in: (i) same-day meals; and, (ii) meals on different days. For the same day, in the breakfast and snack meals, the following preparations are checked: (i) bread/other cereal; (ii) drink; and, (iii) fruit. For different days, in the lunch meal, the same preparation of type (i) entree; (ii) side dish; and, (iii) main dish can be offered only once a week and the same preparation of type (iv) rice, (v) beans, (vi) dessert and (vii) juice can be offered only twice a week.

- Cost limit: The government funds schools in Brazil to provide every child nutrition meals. The value is given by each school day for each student and takes into account the educational level. Thus, the cost of the menu has to be less than the pre-defined cost.

- Lower and Upper Limits of the Macronutrients: The FAO/WHO [17] establishes lower and upper macronutrient limits in relation to energy need. The macronutrient values should be within this established range (Table II).

\section{Modelling of the Problem}

In this Section, the problem formulation is presented. The proposed Genetic Algorithm and its operators are also introduced.

\section{A. Problem Formulation}

In this work, the MPP is formulated using a single-objective approach. The goal is to minimize the cost of a five-day menu while respecting some constraints given by qualitative and quantitative aspects.

The single-objective problem can be formulated as:

$$
\begin{array}{ll}
\text { Minimize } & f=\sum_{d} c_{d} \\
\text { s.a: } \quad & \left\{\begin{array}{l}
\text { Color Constraint } \\
\text { Consistency Constraint } \\
\text { Variety Constraint } \\
\text { Cost Limit Constraint } \\
\text { Lower and Upper Limit of } \\
\text { Macronutrients Constraint }
\end{array}\right.
\end{array}
$$

in which $f$ is the cost function to be minimized, $d$ is a day, and $c_{d}$ is the cost of each day.

Figure 1 represents a possible problem solution representing a menu. In this example, each menu is composed of two days, and for each day, we have meals that are composed by preparations. The meals and preparations are represented by lists as seen in Figure 1(a). Using an integer representation, as shown in Figure 1(b), each type of meal receives a number: (i) Breakfast (1), (ii) Lunch (2); and, (iii) Snack (3). The preparations are also represented by numbers and these numbers refer to the position of the list according to the type of preparation. For each type of preparation, there is a list containing several preparations.

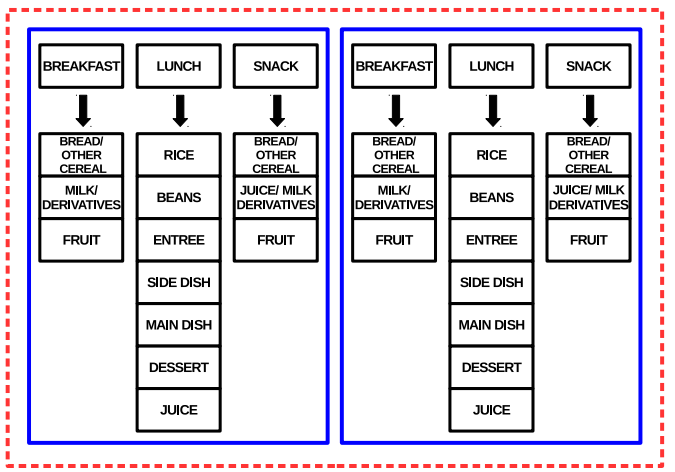

(a) Menu Representation

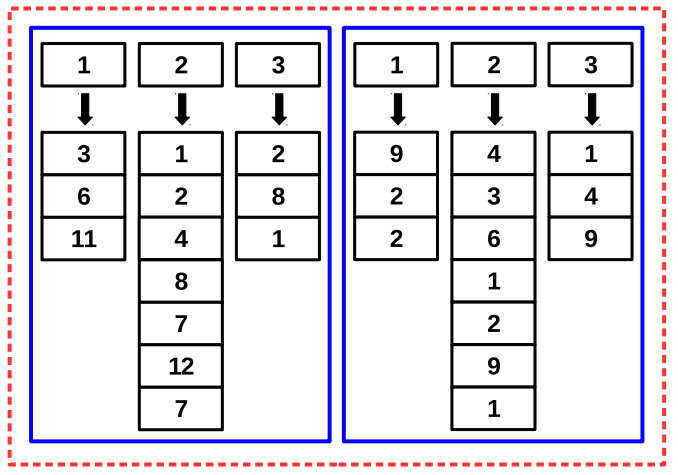

(b) Integer Menu Representation

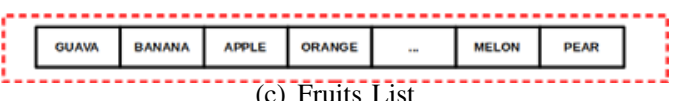

(c) Fruits List

Figure 1. Example of representation of a two-day menu: The last position of the list 1 corresponds to breakfast in Figure 1(b). The number 11 refers to the index of a list of fruits with eleven options. In this case, this index represents a Pear (Figure 1(c)).

\section{B. Genetic Algorithms}

Genetic Algorithms (GA's), introduced by John Holland in 1960 [18], are computational methods of search based in the mechanism of genetic and natural evolution. In a GA's, a set of candidate solution (population) evolves according to probabilistic rules inspired by the natural selection process in which the fittest individuals are selected for the next population. After an initial population is randomly generated, the algorithm evolves through the three operators: selection, crossover, and mutation. Genetic Algorithms, like any other evolutionary technique, are especially well tuned for solving a wide class of problems due to their ability to explore vast solution spaces and search from a family of candidate solutions rather than from just a single point. Algorithm 1 shows a pseudo-code of a basic Genetic Algorithm.

A good initial population plays an important role in the GA's evolution process helping not only to decrease the time to achieve an acceptable final result but also to increase the quality of the final result. Having that in mind, the initial population of the proposed algorithm is generated randomly however some solutions, generated using other heuristics, are included in this initial population. During the random process 
for generating the initial population, depending on the type of meal, random values between zero and the size of the list belonging to that type are generated. As an example, since breakfast consists of a bread/other cereal, a fruit, and a drink, three random values are generated according to the size of each list. These values represent the position in the corresponding list. Then the content of that position is added to the breakfast list. The same process is applied to other types of meal.

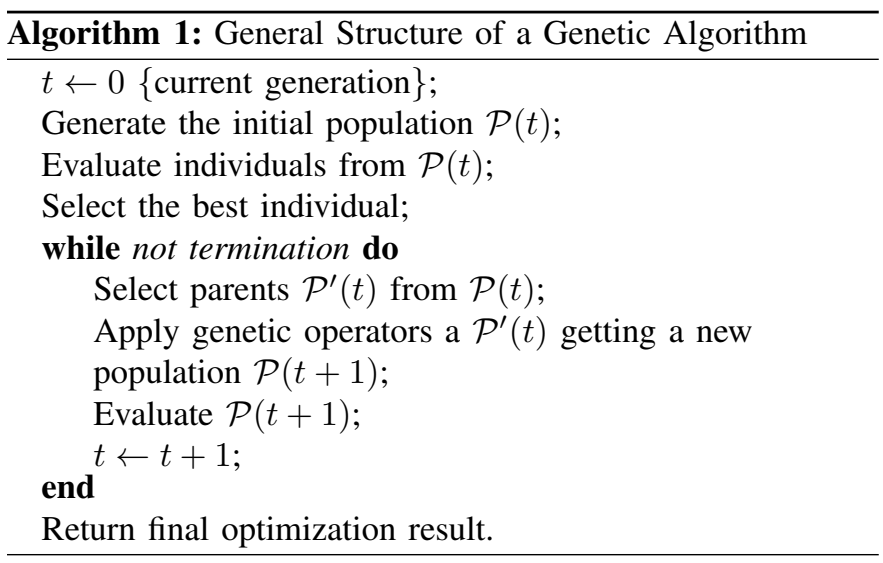

The genetic operators, selection, crossover, and mutation, are defined as follows:

- Selection: the selection is a tournament selection in which two random individuals are chosen and, the one with the best function value is selected.

- Crossover: at each generation, each individual has a chance to be selected for crossover according to the crossover probability. The crossover operator can be performed using various approaches. In this work, it is performed only within each type of meal, according to Figure 2. Considering the crossover of breakfast, and snack, the cutoff point ranges from one to three and, lunch, the cutoff points ranges from one to seven. Thus new individuals are generated preserving the genetic inheritance of its parents.

- Mutation: the mutation chooses a random day of the menu, and for each meal of this specific day, also chooses randomly a preparation to be changed by another one of the same type (Figure 3). At each generation, every individual has a chance to mutate according to a mutation rate and the preparation only be exchanged for another of the same type. For example, one fruit can only be exchanged for another fruit. Thus, in a menu, 3 exchanges can be performed.

Since GA's are unconstrained methods by nature, the constraints are handling via a penalization approach. The color and consistency constraints are modeled using a linear function while the variety constraint is modeled using a quadratic function. This difference is applied in order to enforce a higher penalty factor for the variety. The linear and quadratic functions are then appended to the cost function to form an augmented cost function.

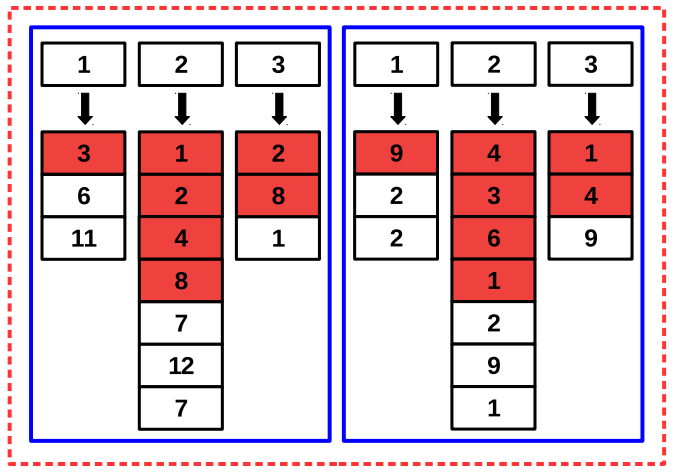

(a) Parent 1

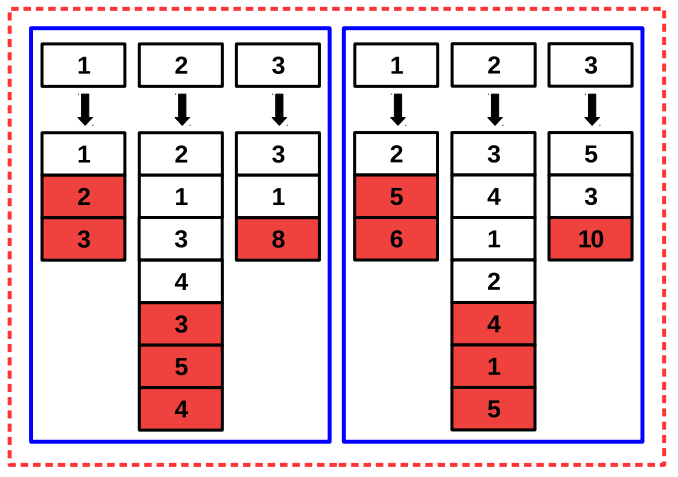

(b) Parent 2

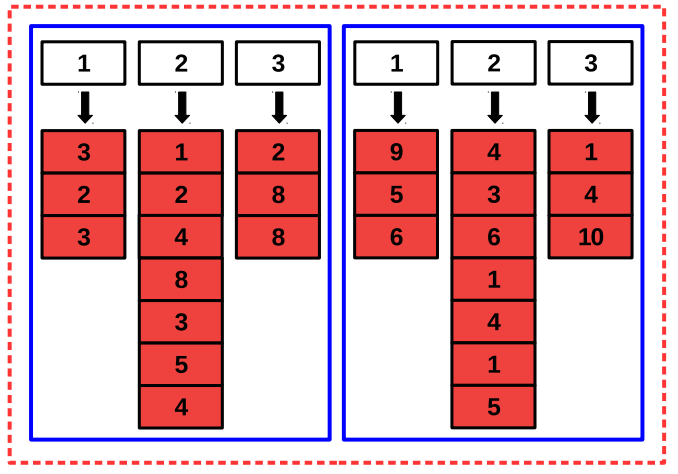

(c) Offspring 1

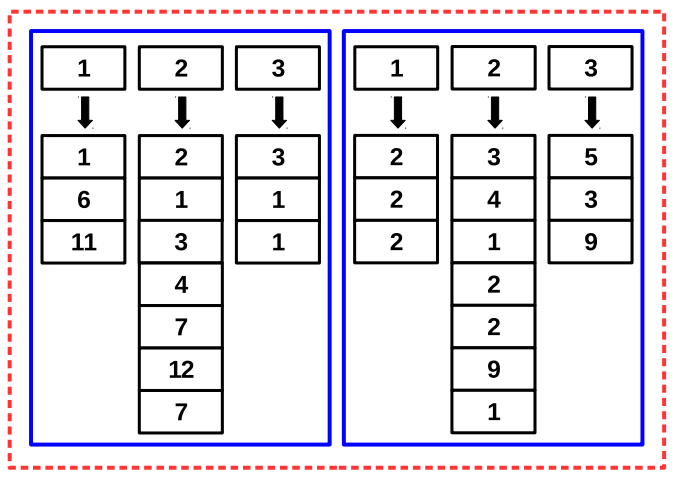

(d) Offspring 2

Figure 2. Example of crossover: Offspring 1 gets from parent 1 preparations from the first preparation of each meal to the cut-off points $(1,4$, and 2$)$ and the cut-off points to the end of parent 2. Offspring 2 gets preparations from parent 2 of the first preparation of each meal to the cut-off points $(1,4$, and 2) and the cut-off points to the end of parent 1 . 


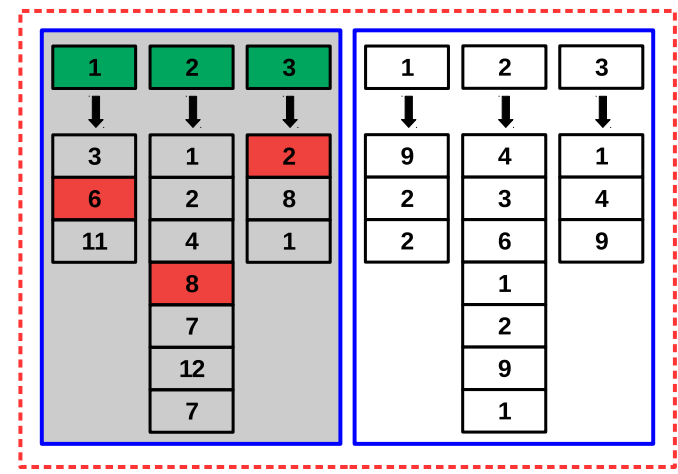

(a) Before mutation

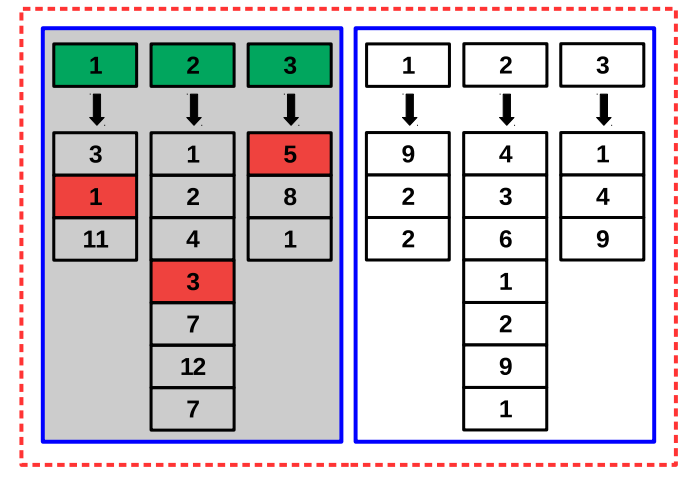

(b) After mutation

Figure 3. Example of a mutation that exchanges, in the breakfast, the preparation 6 by 1 of the milk or derivatives; In the lunch, the mutation exchanges the preparation 8 by 3 of the side dish; In the snack meal, preparation 2 is exchanged by 5 of the bread/ other cereal on the first day of a 2-day menu.

\section{Results}

The proposed GA's was used to solve the single-objective version MPP. The five-day menu was composed by three meals daily. The limit values of the meals were fixed at: breakfast: $\mathrm{R} \$ 2.00$, lunch: $\mathrm{R} \$ 4.00$, and snack: $\mathrm{R} \$ 2.00$. The age group considered was 4 to 5 years, equivalent to the full-time preschool $(B R L-R \$ 1.00 \approx U S D-\$ 0.31)$. The algorithm was executed 30 times and was started with the same basic parameters as listed below:

- population size: 100 individuals

- crossover probability: 0.8

- mutation probability: 0.05

- maximum number of generations: 1000

All parameters are within the usual range however they were chosen empirically and following the setting presented at [14]. For a discussion of the parameter effect over the GA's performance, an exhaustive investigation will be carried out in a future work.

The maximum number of generations was the only stopping criterion. At the end of 30 algorithm runs, we obtained the mean convergence line for the GA's. The mean convergence line corresponds to the mean value of the best individual throughout the 1000 generations. Figure 4 presents the mean convergence line of the algorithm.

Analyzing Figure 4, it is possible to see that the algorithm needed time to converge. The algorithm stabilized at some points and, later on, found better solutions. For example, some plateau can be seen between generations 324 to 452 and 494 to 761 . After 762 generations, small improvements in the objective function value can be seen. However, the final objective function value was $\mathrm{R} \$ 14.8736$, a smaller value than the pre-defined cost of $\mathrm{R} \$ 40.00$. The algorithm generated a menu $62 \%$ cheaper than the maximum limit allowed. The obtained menu also respected all the established constraints.

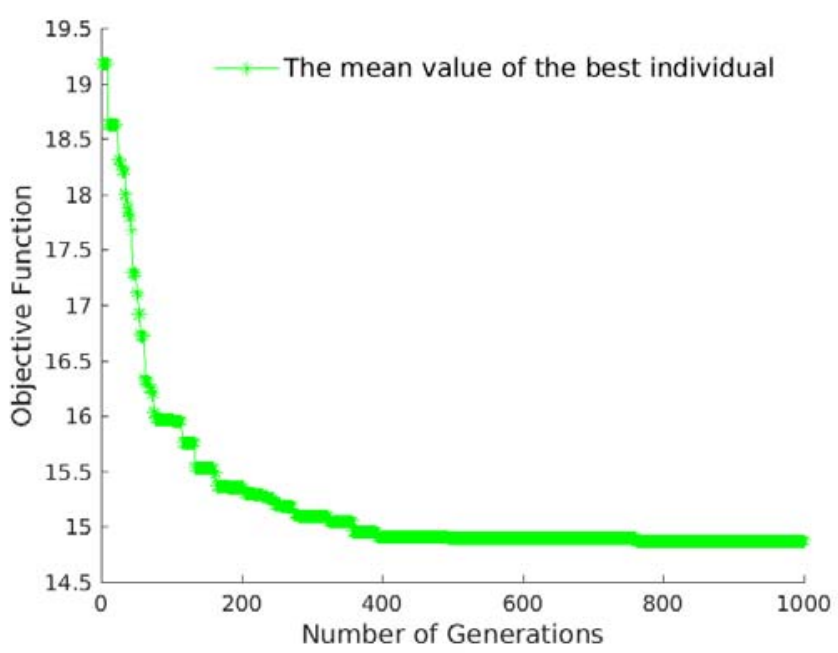

Figure 4. The convergence line corresponds to the mean value of the best individual found in 30 runs of the GA's throughout the 1000 generations. The $\mathrm{x}$-axis represents the generations and the $\mathrm{y}$-axis represents the objective value of the best individual.

Figure 5 present the five-day menu given by the Genetic Algorithm. There are some significant aspects we need to notice. The total cost limit and the qualitative and quantitative constraints were respected. Besides, keeping the macronutrients under a pre-defined range, it was possible to attend the pre-established micronutrients levels without the need to include it in the problem formulation.

A comparison of the obtained menu with previous solutions needs to be carried out. However, in [14], the formulation of the nutritional error calculates the difference between the total of macro and micronutrients and the pre-defined ones. In this work, the formulation aims to target the macronutrients into a pre-established range. In that way, there is a lack of suitable previous approaches to perform a fair comparison. Aiming at providing a comparison of the proposed approach, a greedy-based procedure was applied to create menu having the same goal and same set of constraints. As shown previously, each solution is composed by meals and each meal is composed by preparations. A list of the available preparations for composing a meal is evaluated and sorted, in ascending 


\begin{tabular}{|c|c|c|c|c|c|}
\hline Meal & Day 1 & Day 2 & Day 3 & Day 4 & Day 5 \\
\hline \multirow[t]{3}{*}{ Breakfast } & Banana Cake & Salt Cracker & Carrot Cake & Nutritive Cake & Cookie \\
\hline & $\begin{array}{l}\text { Chocolate } \\
\text { Milk }\end{array}$ & $\begin{array}{l}\text { Coffee with } \\
\text { Milk }\end{array}$ & $\begin{array}{l}\text { Chocolate } \\
\text { Milk }\end{array}$ & $\begin{array}{l}\text { Coffee with } \\
\text { Milk }\end{array}$ & $\begin{array}{l}\text { Caramelized } \\
\text { Milk }\end{array}$ \\
\hline & Tangerine & Pineapple & Orange & Banana & Banana \\
\hline \multirow{7}{*}{ Lunch } & White Rice & $\begin{array}{c}\text { Broccoli- } \\
\text { Chicken Rice }\end{array}$ & $\begin{array}{l}\text { Rice with } \\
\text { Lentil }\end{array}$ & White Rice & $\begin{array}{c}\text { Broccoli- } \\
\text { Chicken Rice }\end{array}$ \\
\hline & Mashed Beans & Mashed Beans & Plain Beans & $\begin{array}{c}\text { Meat Sauce } \\
\text { Beans }\end{array}$ & Plain Beans \\
\hline & $\begin{array}{c}\text { Spring Greens } \\
\text { Salad }\end{array}$ & Carrot salad & $\begin{array}{c}\text { Tomato/ } \\
\text { Cabbage Salad }\end{array}$ & $\begin{array}{l}\text { Spring Greens/ } \\
\text { Tomato Salad }\end{array}$ & Beet and Carrot \\
\hline & $\begin{array}{l}\text { Chicken/Potato } \\
\text { Puree }\end{array}$ & $\begin{array}{l}\text { Steamed } \\
\text { pumpkin }\end{array}$ & $\begin{array}{c}\text { Spinach/ } \\
\text { Cornmeal Cream }\end{array}$ & $\begin{array}{c}\text { Banana/ } \\
\text { Chicken Stew }\end{array}$ & $\begin{array}{c}\text { Sweetcorn/ } \\
\text { Pumpkin Puree }\end{array}$ \\
\hline & Chicken Kibbeh & Battered Fish & $\begin{array}{c}\text { Mince } \\
\text { meat/okra }\end{array}$ & $\begin{array}{l}\text { Chicken } \\
\text { Stew }\end{array}$ & $\begin{array}{l}\text { Meatballs } \\
\text { In Sauce }\end{array}$ \\
\hline & $\begin{array}{c}\text { Caramelized } \\
\text { Banana }\end{array}$ & Pineapple & Papaya & Papaya & $\begin{array}{c}\text { Caramelized } \\
\text { Banana }\end{array}$ \\
\hline & Grape Juice & Grape Juice & Cashew Juice & Orange Juice & Lemon Juice \\
\hline \multirow[t]{3}{*}{ Snack } & Nutritive Cake & Banana Cake & Salt Cracker & Sweet Cookie & Carrot Cake \\
\hline & Orange Juice & $\begin{array}{l}\text { Caramelized } \\
\text { Milk }\end{array}$ & Cashew Juice & $\begin{array}{l}\text { Passion Fruit } \\
\text { Juice }\end{array}$ & Lemon Juice \\
\hline & Orange & Watermelon & Watermelon & Melon & Melon \\
\hline \multicolumn{6}{|c|}{ Total Cost: $\mathrm{R} \$ 14.8736$} \\
\hline
\end{tabular}

(a) The 5-day Menu

\begin{tabular}{|l|c|c|c|c|c|c|c|c|}
\hline & CHO & PTN & LIP & Fibers & \multicolumn{3}{|c|}{ Minerals $(\mathrm{mg})$} \\
\cline { 2 - 10 } Reference & $\begin{array}{c}\text { (g) } \\
\begin{array}{c}130.6250 \\
\text { to } \\
178.1250\end{array}\end{array}$ & $\begin{array}{c}23.7500 \\
\text { to } \\
35.6250\end{array}$ & $\begin{array}{c}15.8333 \\
\text { to } \\
31.6667\end{array}$ & 17.5000 & 560.0000 & 91.0000 & 7.0000 & 3.5000 \\
\hline Day 1 & 177.2551 & 34.9375 & 29.1760 & 14.1731 & 351.4220 & 181.8850 & 5.1225 & 3.2249 \\
\hline Day 2 & 168.3439 & 35.5522 & 24.6483 & 15.1721 & 437.2318 & 194.7037 & 4.9654 & 3.8233 \\
\hline Day 3 & 155.0816 & 26.9507 & 28.6350 & 16.9926 & 356.3758 & 193.8887 & 6.2595 & 4.8364 \\
\hline Day 4 & 160.0707 & 30.1101 & 27.7861 & 14.4294 & 330.1989 & 191.6239 & 6.8305 & 3.9529 \\
\hline Day 5 & 166.2500 & 32.8576 & 30.2815 & 14.4142 & 268.7517 & 177.6155 & 4.6933 & 4.0272 \\
\hline
\end{tabular}

(b) Nutritional values

Figure 5. Figure 5(a) shows the best 5-day menu with breakfast, lunch and snack found by algorithm and Figure 5(b) shows the nutritional values of this menu and the established nutritional reference. The reference of macronutrients (CHO, PTN, LIP) presented consider their lower and upper limits and the other nutrients consider the PNAE reference.

order, using the following equation:

$$
f_{\text {val }}=0.5 \cdot \operatorname{sum} N+0.5 \operatorname{cost}
$$

in which $\operatorname{sum} N$ is the sum of all normalized macronutrients and cost represents the normalized monetary cost of the menu. In the greedy-based approach, the preparation with the smallest value of function is chosen. If the preparation with the smallest function value is not feasible, the subsequent one is chosen until a feasible preparation is found. A mechanism to preserve the feasibility regarding the number of times a specific preparation is used to compose a meal is included in the approach. When the number of repetitions reaches the limit, the preparation is removed from the preparation list. It is worthwhile to notice that this is a deterministic algorithm. This means that, starting from the same data base, all the menus generated using this approach will be exactly the same.
Table III shows the nutritional values of greedy-approach menu and the established nutritional reference. The reference of macronutrients (CHO, PTN, LIP) presented consider their lower and upper limits and the other nutrients consider the PNAE reference. The total cost of the greedy menu is $\mathrm{R} \$$ 15.9032. Comparing the 5-day menu obtained by the proposed approach 5 and the greedy-approach menu III, the total cost value of both menu are not so different. Since the greedy-based menu was obtained using a constrained approach related to variety, consistency and color, the respective constraints were satisfied. However, in the greedy-based menu, all macronutrients are outside of the pre-defined range at least in one day of the week. The bold values in Table III show the macronutrients outside the range.

For assessing the quality of the obtain menu, an evaluation made by a nutritionist is required. The obtained menu and 
Table III

THE NUTRITIONAL VALUES OF GREEDY-APPROACH MENU AND THE ESTABLISHED NUTRITIONAL REFERENCE. THE VALUES IN BOLD SHOW THE MACRONUTRIENTS WHICH ARE OUTSIDE THE PRE-DEFINED RANGE.

\begin{tabular}{|l|r|r|r|r|r|}
\hline & \multicolumn{1}{|c|}{ DAY 1 } & \multicolumn{1}{c|}{ DAY 2 } & \multicolumn{1}{c|}{ DAY 3 } & \multicolumn{1}{c|}{ DAY 4 } & \multicolumn{1}{c|}{ DAY 5 } \\
\hline ENERGY & 896.4839 & 796.8484 & 1124.9048 & 1199.0724 & 1212.1225 \\
\hline PTN & 27.5497 & $\mathbf{2 1 . 3 9 1}$ & $\mathbf{3 9 . 1 5 4 1}$ & $\mathbf{4 0 . 4 8 4}$ & $\mathbf{4 5 . 4 5 8 3}$ \\
\hline LIP & 23.8385 & 24.0242 & 23.7841 & 29.3371 & $\mathbf{3 4 . 9 6 5 4}$ \\
\hline CHO & 148.3622 & 133.029 & $\mathbf{1 9 7 . 4 8 8 2}$ & $\mathbf{1 9 7 . 4 6 5 3}$ & $\mathbf{1 8 3 . 6 0 8 7}$ \\
\hline FIBER & 13.0263 & 11.0217 & 14.6351 & 15.7027 & 16.7986 \\
\hline CA & 196.6117 & 205.8709 & 341.4127 & 359.2742 & 509.6139 \\
\hline MG & 155.1389 & 145.7376 & 190.0684 & 182.6793 & 239.6612 \\
\hline FE & 4.2908 & 4.1782 & 6.561 & 5.924 & 5.2174 \\
\hline ZN & 2.7684 & 2.9417 & 6.6319 & 3.8128 & 4.5603 \\
\hline
\end{tabular}

their nutritional values, represented by Figure 5, was sent to an experienced nutritionist. According to the nutritionist feedback, the menu meets the PNAE recommendations. Even considering only macronutrient limits, the micronutrients were close to the reference ones. The menu is suitable to be used in the school environment. However, there are some rooms for improving the menus. Repetitions of flavors on the same day, such as orange cake and orange juice, should be avoided. Although repetitions in consecutive days are allowed, it would be recommended to avoid this practice. A positive aspect that must be noticed relies on the time required to generate the menu. An automated way to generate five-days menu spending 60 seconds could facilitate the nutritionist work.

\section{CONCLUSIONS}

This paper presented a single-objective formulation for the Menu Planning Problem. The goal was to plan low-cost menus in an automatic and diversified manner while satisfying the requirements established by the government via the Brazilian School Eating Programme (PNAE) guidelines. The single-objective problem was solved using a Genetic Algorithm. Results showed that the proposed approach was able to generate menu within the pre-defined cost value and the requirement constraints were respected. A comparison between the obtained 5-day menu and a constrained greedy-based approach was carried out. Despite the fact the obtained menu outperforms the greed-based menu taking into account the total cost, this difference is not so expressive. However, all macronutrients were outside the pre-defined range at least in one day of the week. A nutritionist assessed the obtained menu. The overall quality of the menu was outstanding and the time spent to generate it was 60 seconds. Some additional constraints and recommendation can be taken into account in future works.

\section{ACKNOWLEDGMENT}

This work was partially funded by CEFET-MG, CNPq, FAPEMIG, CAPES and SARI, Aston University.

\section{REFERENCES}

[1] C. J. L. Murray, M. N. Fleming, E. Gakidou, and M. Robinson, "Global, regional, and national prevalence of overweight and obesity in children and adults during 1980-2013: a systematic analysis for the global burden of disease study 2013," The Lancet., no. 14, 2014.

[2] R. Dobbs and J. Manyika, "The obesity crisis," Cairo Review, July 2015.

[3] WHO, "World health organization. levels and trends in child malnutrition," may 2017.

[4] IBGE, Synthesis of Social Indicators - An analysis of the living conditions of the Brazilian population. 2013.

[5] Brazil, "Ministry of social development and fight against hunger.food security will be a priority in the pluriannual plan 2016-2019," jun 2015.

[6] Brazil, "Ministry of education. national fund for the development of education (fnde) pnae: School feeding," 2017.

[7] J. Balintfy, "Menu planning by computer," Comunications of the ACM, vol. 7, pp. 255-259, April 1964.

[8] B. Gaal, I. Vassányi, and G. Kozmann, "A novel artificial intelligence method for weekly dietary menu planning," Methods Inf Med, pp. 655-664, May 2005.

[9] A. Kahraman and H. A. Seven, "Healthy daily meal planner," Genetic and Evolutionary Computation Conference - Undergraduate Student Workshop (GECCO 05 UGWS), pp. 25-29, 2005.

[10] J. Bulka, A. Izworski, J. Koleszynska, J. Lis, and I. Wochlik., "Automatic meal planning using artificial intelligence algorithm in computer aided diabetes therapy," IEEE, pp. 393-397, February 2009.

[11] B. K. Seljak, "Comput-based dietary menu planning," Journal of Food Composition an Analysis, pp. 414-420, 2009.

[12] F. R. Gomes, "Pro-diet: Automatic generator of personalized menus based on genetic algorithm," masters dissertation, Federal University of Uberlândia, Uberlândia, 2012.

[13] A. R. Cruz, T. G. Lima, M. F. Araújo, and N. R. C. Cruz, "Uma abordagem multiobjetivo inteira para a dieta em creches," Anais do XLV Simpósio Brasileiro de Pesquisa Operacional, 2013.

[14] R. P. C. Moreira, E. F. Wanner, F. V. C. Martins, and J. F. M. Sarubbi, "The menu planning problem: a multiobjective approach for brazilian schools context," in Genetic and Evolutionary Computation Conference, Berlin, Germany, July 15-19, 2017, Companion Material Proceedings, pp. 113-114, 2017.

[15] M. Garey and D. Johnson, Computers and Intractability: A Guide to the Theory of NP-Completeness. Series of books in the mathematical sciences, W. H. Freeman, 1979.

[16] FAO, "Food and agriculture organization of the united nations," 2017.

[17] WHO, "World health organization. diet, nutrition and the prevention of chronic diseases," tech. rep., WHO Library Cataloguing-in-Publication Data, 2003

[18] J. H. Holland, Adaptation in Natural and Artificial Systems. Cambridge, Massachusetts, 1975 